\title{
Predictive Control Applied to a Cascaded H-Bridge Multilevel Converter
}

\author{
Mohammad Ali Hosseinzadeh ${ }^{1}$, Maryam Sarbanzadeh ${ }^{1}$, Elham Sarbanzadeh ${ }^{2}$, Marco Rivera $^{1}$ and Patrick Wheeler ${ }^{3}$ \\ ${ }^{1}$ Faculty of Engineering, Universidad de Talca, Curico, Chile \\ m.a_hosseinzadeh@yahoo.com; maryam_sarbanzadeh@yahoo.com; marcoriv@utalca.cl \\ ${ }^{2}$ Faculty of Engineering, University of Tabriz, Tabriz, Iran \\ el_sarebanzade@yahoo.com \\ ${ }^{3}$ Faculty of Engineering, The University of Nottingham, UK \\ Pat.Wheeler@nottingham.ac.uk
}

\begin{abstract}
Power converters are the main part of power electronic applications. A predictive strategy is a new control strategy to control cascaded H-Bridge (CHB) converter due to the control of multivariables, simultaneously. This report presents an overview on recent advanced predictive control techniques applied to a cascaded $\mathrm{H}$-bridge (CHB) multilevel converter. The selected papers are chosen from the IEEE-explore database which have been published in the last couple of years.

Index Terms-predictive control, cascaded h-bridge converter, mathematical model.
\end{abstract}

\section{INTRODUCTION}

The conventional multilevel converters-inverters (MLCs/MLIs) configurations with industrial applications include cascaded H-bridge (CHB), a flying capacitor (FC) and natural diode clamped (NPC) multilevel converters [1]. The CHB converter is formed by H-bridge cells series connection and each cell is connected by dc isolated supplies. The CHB converter based on DC isolated supplies values is separated into two groups of symmetrical and asymmetrical structures. The magnitudes of the whole input of dc supplies are equal to the structure however in an asymmetrical one they are dissimilar. This report focuses on a three-phase cascaded H-bridge converter in the symmetrical mode, to apply the voltage for an R-L load [2]. Generally, there are three strategies in order to control and modulate a $\mathrm{CHB}$ converter: linear control, pulse width modulation (PWM) (carrier based) [3] and space vector modulation (SVM) [4], [5]. Other control strategies for low switching frequency have been suggested in [6]-[8].

New control methods have been introduced for power converters in the last decades. The presented method is the Model Predictive Control (MPC) which is used in the control of power converters due to ease of non-linear access, fast dynamic response and system constraints [9]-[11]. The MPC considers a system model to estimate its next behavior at a time horizon. In this case, to obtain the optimisation of the system's behavior a cost function is defined. On the other hand, model predictive control solves the optimisation issue through a string of future actions which are achieved by minimizing the cost function. All calculations are done sequentially in each instance period and the first component of the string is implemented.

In this report, a review on recent predictive control schemes for a $\mathrm{CHB}$ converter is presented. First, a mathematical model of the CHB converter is illustrated in detail. Next, the principle of predictive control applied to it is discussed. Finally, recent research papers that have been presented on the IEEE-explore database for $\mathrm{CHB}$ converters are reviewed, which have been published in the last couple of years. It is expected that the papers will contribute to all researchers that are starting to work in multilevel application areas.

\section{CHB Converter Mathematical Model}

Fig. 1 displays the power topology of a three-phase CHB converter. This configuration includes two CHBs in each phase [12]. Each CHB creates 3 voltage levels of $\pm V_{d c}$ and 0. Therefore, for each phase, the number of voltage levels is as follows:

$$
N_{L}=2 n+1
$$

Each cell in each phase is controlled by two independent switching functions and the a-phase cell voltage $V_{a i}$ is calculated as:

$$
V_{a i}=V_{d c}\left(S_{1 a, i}-S_{2 a, i}\right)
$$

As a result, the output voltage of the converter in to the neutral point $N$ includes the total of output voltages of each cell. The $\mathrm{V}_{a N}$ is obtained by:

$$
V_{a N}=\sum_{i=0}^{n} V_{a i}
$$

from the equations of (2) and (3), $\mathrm{V}_{a N}$ can be expressed as:

$$
V_{a N}=V_{d c} \sum_{i=0}^{n}\left(S_{1 a, i}-S_{2 a, i}\right)
$$

The number of switching states $K_{S}$ for an $n$ cell of the CHB converter in each phase is obtained:

$$
K_{S}=2^{6 n}
$$




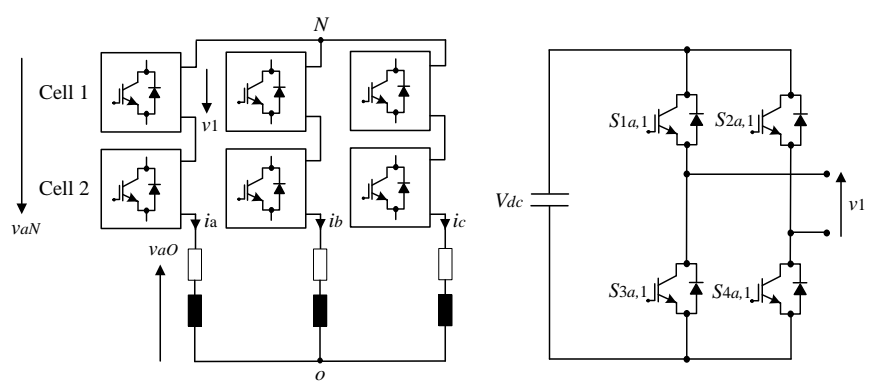

Fig. 1: Two-cell CHB three-phase inverter.

Generally, the total of switching states in a 3-phase CHB converter is 4096.

This number of switching states is highly satisfying. In this way, in a 2-cell $\mathrm{CHB}$ converter, each phase creates 5 voltage levels, therefore the sum of the voltage vectors are 125 . Due to the fact that several redundant states exist, the total different voltage vectors will decrease to 61 .

\section{Principle of Predictive Control In CHB CONVERTERS WITH R-L LOAD}

The predictive current control uses the switching state selection of the converter for providing the controlled variables to the desired references. In this control method, the objective is to the next treatment of variables upon a time structure that uses multiples of the sample time. A simple load model and the relation of it to the output voltage can be expressed as:

$$
\begin{gathered}
v_{a o}=R i_{a}+L \frac{d i_{a}}{d t} \\
v_{a o}=v_{a N}+v_{N o}
\end{gathered}
$$

where $v_{a o}$ is the load voltage. Considering the Clarcke transformation:

$$
\left[\begin{array}{l}
\alpha \\
\beta
\end{array}\right]=\left[\begin{array}{ccc}
\frac{2}{3} & -\frac{1}{3} & -\frac{1}{3} \\
0 & \sqrt{\frac{3}{3}} & -\sqrt{\frac{3}{3}}
\end{array}\right]\left[\begin{array}{l}
a \\
b \\
c
\end{array}\right]
$$

where $\alpha$ and $\beta$ are the vector variables and the 3-phase system variables are $a, b$, and $c$. Therefore, the relation of (6) will be rewritten as follows:

$$
v_{\alpha, \beta}=L \frac{d i_{\alpha, \beta}}{d t}+R i_{\alpha, \beta}
$$

where $i_{\alpha, \beta}$ and $v_{\alpha, \beta}$ are the load current and the inverter's voltage vector, respectively. Considering the definition of the derivative and substituting in (9), we will have:

$$
i_{\alpha, \beta}=\frac{T_{s}}{L}\left(v_{\alpha, \beta}[k]-i_{\alpha, \beta}[k]\left(R-\frac{L}{T_{s}}\right)\right)
$$

The current following error is a key variable in current control methods. Therefore, the simplest and most straightforward option for the cost function is the absolute value of the current error. Other options are the square error, the current error integral, and the changing of the rate of error. For the square current error, the cost function will be as follows:

$$
g[k+1]=\left|i_{\alpha}^{*}[k+1]-i_{\alpha}[k+1]\right|+\left|i_{\beta}^{*}[k+1]-i_{\beta}[k+1]\right|
$$

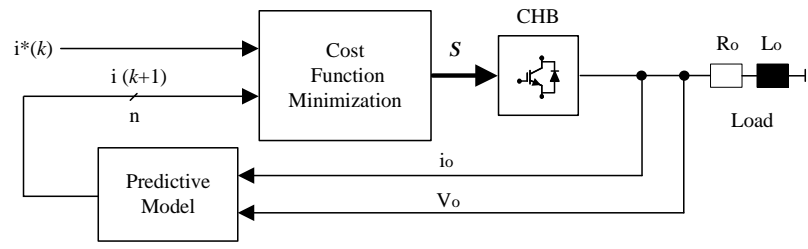

Fig. 2: Predictive control diagram for $\mathrm{CHB}$ inverter.

where $i_{\alpha, \beta}^{*}[k+1]$ is the reference current vector prediction. By assuming $i_{\alpha, \beta}^{*}[k+1] \approx i_{\alpha, \beta}^{*}[k]$ makes longer small sampling times. Extraction of the reference is essential for larger sampling time.

The cost function is calculated for each voltage vector and the smallest value is chosen. Namely, the relations of (11) and (12) are computed 125 times for a five-level converter to achieve the most adequate solution. Fig. 2 displays the predictive current control scheme. In this state, considering $n$ number of voltage vectors, the monitored currents are applied to estimate $n$ future values of the load. Finally, by the cost function, the predictions are estimated and the voltage vector that minimises the function is chosen.

\section{Recent Predictive Control Method applied to CHB CONVERTERS}

Based on the review done in [13]-[15], the CHB converter is utilized as a static synchronous compensator (STATCOM) and an FCS-MPC is employed to control the CHB converter. In a cascaded h-bridge converter applied to a STATCOM system, the components of the verification objects are the plot of the reference current, the balance of the capacitors' voltage balance, and optimisation of the switching frequency. In this case, by defining a cost function and the weighting of the coefficients, the total of the control objects is evaluated.

The finite control set-MPC standard strategy for cascaded h-bridge STATCOM is displayed in Fig. 3. As one can note, this strategy contains the reference current production and a finite control set-MPC. The reference current consists of two active and reactive components so that the first is obtained from the general external voltage loop [16], and the latter is specified by the demand for compensation of the reactive power. Considering the sampling and calculation delay, the model predictive control should be applied in a two-step estimation approach [17].

In this way, extrapolation and prediction cycles are necessary. At the sampling time of $t=k T_{s}$, suppose that $i_{\alpha, \beta[k]}^{*}$ is the reference currents and $V_{C(k)}^{*}$ is the reference voltage of capacitor. To hypothesis the voltage of the capacitors and reference currents, the hypothesized cycle is used at $t=(k+2) T_{s}$. By the switching states given in the final sampling cycle and sample values, the prediction cycle is applied to predict the grid current and voltage at $t=(k+1) T_{s}$. Therefore, the relation of prediction currents and voltages of the capacitors 
will be:

$$
\begin{gathered}
i_{\alpha, \beta(k+2)}^{P}=\hat{i}_{\alpha, \beta(k+1)}+\left(\hat{v}_{s \alpha, \beta(k+1)}-R \hat{i}_{\alpha, \beta(k+1)} v_{\alpha, \beta(k+1)}\right) \frac{T_{s}}{L} \\
v_{c(p i)(k+2)}^{p} \approx v_{C(p i)(k)}+s_{(p i)(k+1)} i_{p(k+1)} \frac{T_{s}}{C}
\end{gathered}
$$

Therefore, the cost function in a standard finite control setMPC is determined as:

$$
\begin{array}{r}
J\left(s_{p i(k+1)}\right)=\lambda_{c a p} \sum_{1}^{n}\left\|v_{C(k+2)}^{r e f} e-v_{C p i(k+2)}^{p}\right\|+ \\
\left\|i_{\alpha, \beta(k+2)}^{r e f}-i_{\alpha, \beta(k+2)}^{p}\right\|+\lambda_{\text {switch }} \sum_{1}^{n}\left\|s_{p i(k+1)}-s_{p i(k)}\right\|
\end{array}
$$

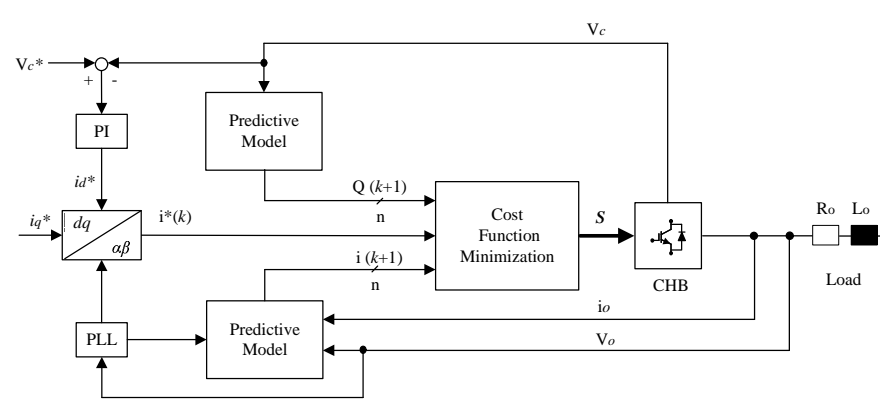

Fig. 3: FCS-MPC Standard scheme for STATCOM based on CHB inverter.

Considering (14), the aim of each term is, respectively, to set the voltage of the capacitors, set the current, limit the switching attempts, and $\lambda_{\text {switch }}$ is the weighting factor. To express the error between the reference and the expected value a rule function is used, which is chosen from 1 to $\infty$-norm.

Considering (14), the cost function is specified through $J\left(s_{p i(k+1))}\right.$. By selecting the switching mixture which can minimize $J\left(s_{p i(k+1)}\right)$, it is possible to obtain the control objectives.

A fast MPC diagram for a CHB based on STATCOM has been presented in [13] for solving the problem of an exponentially increasing computational burden of an FCSMPC. The presented MPC can find the overall best outcome by applying the polynomial subject to time. This is obtained by the three subsequent ways. First, a laminated optimisation procedure is adjusted to separate the standard model predictive control into a model predictive control current and model predictive control of balancing voltage of the capacitor. The current control cost function is expressed as:

$$
\begin{aligned}
J 1\left(s_{\alpha, \beta(k+1)}\right)= & \left\|i_{\alpha, \beta(k+2)}^{r e f}-i_{\alpha \beta(k+2)}^{p}\right\|+ \\
& \lambda_{s 1}\left\|S_{\alpha, \beta(k+1)}-S_{\alpha, \beta(k)}\right\|
\end{aligned}
$$

Thus, the cost function to balance the capacitor voltage and to optimise the switching frequency is explained as:

$$
\begin{array}{r}
J 2\left(s_{p i(k+1)}\right)=\sum_{1}^{n}\left\|v_{C(k+2)}^{r e f}-v_{C p i(k+2)}^{p}\right\|+ \\
\lambda_{s 2} \sum_{1}^{n}\left\|s_{p i(k+1)}-s_{p i(k)}\right\|
\end{array}
$$

In the second one, to detect the best outcome of the model predictive control current, a dynamic decoding algorithm is used. In this approach, considering (15), the relation of $J 1\left(S_{\alpha \beta(k+1)}\right)$ is defined as:

$$
\left\{\begin{array}{l}
J 1\left(S_{\alpha \beta(k+1)}\right)=J_{\alpha(k)}+J_{\beta(k)} \\
J_{\alpha(k)}=\left\|i_{\alpha(k+2)}^{r e f}-i_{\alpha(k+2)}^{p}\right\|+\lambda_{s 1}\left\|S_{\alpha(k+1)}-S_{\alpha(k)}\right\| \\
J_{\beta(k)}=\left\|i_{\beta(k+2)}^{r e f}-i_{\beta(k+2)}^{p}\right\|+\lambda_{s 1}\left\|S_{\beta(k+1)}-S_{\beta(k)}\right\|
\end{array}\right.
$$

Third, a diverse optimisation algorithm has been developed to solve the best switching mixture in balancing the capacitors' voltage. Reduction of the calculation volumes to polynomial time creates the model prediction control which is employed online for a cascaded h-bridge-STACOM. In this case, the running time of the finite control set-MPC has decreased the exponential level to a multinomial level, additionally, when the voltage of the capacitor is well balanced it achieves the overall optimisation outcome. The output current waveform with this method is approximately identical (via a lower calculation time) to the standard overall optimisation strategy. In [14], an easy branch and bound method has been presented to attempt a reduction of the calculation of the cascaded h-bridgeSTATCOM. In this method, the main idea is founded on the branch and bound strategy. The presented method can decrease the calculation volume of a current prediction control cycle to a linear time horizon. The presented method in [14] contains three stages: initially, the optimisation issue in the current predictive control cycle is changed to a two-variable integer decoding criterion with traditional $60 \mathrm{gh}$ peculiarities. [18]. The transformation among the $g h, a b c$ and $\alpha \beta$ is provided as:

$$
\begin{aligned}
& {\left[\begin{array}{l}
S_{g} \\
S_{h}
\end{array}\right]=\left[\begin{array}{ccc}
1 & -1 & 0 \\
0 & 1 & -1
\end{array}\right]\left[\begin{array}{l}
S_{a} \\
S_{b} \\
S_{c}
\end{array}\right]} \\
& {\left[\begin{array}{l}
S_{\alpha} \\
S_{\beta}
\end{array}\right]=\sqrt{\frac{2}{3}}\left[\begin{array}{cc}
1 & \frac{1}{2} \\
0 & \sqrt{\frac{3}{2}}
\end{array}\right]\left[\begin{array}{l}
S_{g} \\
S_{h}
\end{array}\right]}
\end{aligned}
$$

In the second stage, after choosing the value of one variable as a branch, for every branch, a programming problem of a single-variable integer is formulated. Finally, the overall outcome is achieved by the re-count of the best integer outcome of each single-variable integer decoding issue. Thus, the number of values of the branch with regard to the series steps is linear, the calculation complication of the presented method is a linear time level. On the other hand, the presented algorithm finds the optimal overall results. In [15], a new predictive modulation strategy has been developed that distributes losses equally between semiconductors, balances capacitor voltages, 
and produces output voltage with a high spectral quality waveform. This strategy switches an $\mathrm{H}$-bridge during each confirmation step to deploy the required voltage to step deeply into the capacitor's bridge voltage and the loss condition to the semiconductor components. Modulation provides significant computational models that supply a significant method for the regulation of the capacitor's voltage and loss distributions between the units of the cascaded converter. The presented control method for the CHB-STATCOM is displayed in Fig. 4. In order to compute the needed output voltage CHBSTATCOM based on the dead-beat equations, are given as follows:

$$
\begin{gathered}
v_{k+1}^{r e f}=\frac{L}{T}\left(i_{k+1}^{r e f}-\hat{i}_{k}\right)+\hat{v}_{k+0.5}^{\text {sys }} \\
\hat{i}_{k}=i_{(k-1)}+\frac{T}{L}\left(v_{k}-\hat{v}_{k+0.5}^{s y s}\right)
\end{gathered}
$$

where, the value of the reference current is $k \in Z, i_{k+1}^{r e f}$ and the instantaneous sample of the current is $i_{k-1}$.

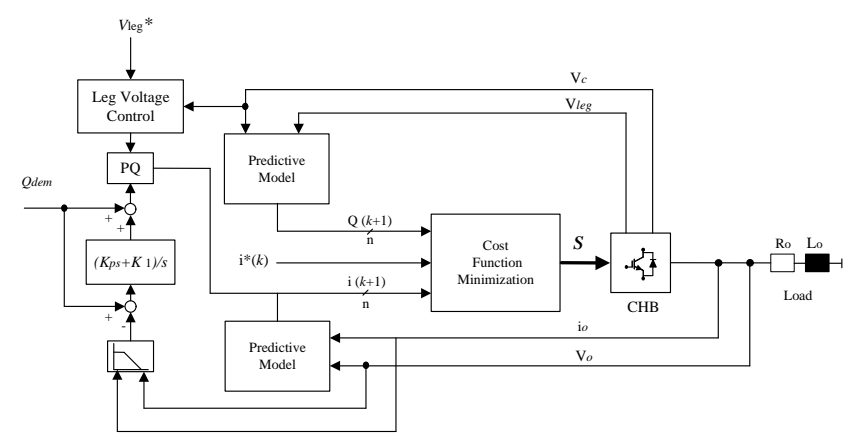

Fig. 4: Presented MPM scheme to control H-STATCOM in [15].

For combining every phase demanded in the output voltage, the model predictive modulation block is accountable. This approach exerted two adjoining voltage levels into every verification cycle for every phase. In order to obtain the voltage reference time integral, they are waited by time.

Then, the algorithm attempts to distribute switching losses depending on which semiconductors have incurred the most/least total loss. By applying the subsequent specification, each leg is assessed. If the error related to $\operatorname{Err}_{l e g, j}=i n f$ considering that a leg has been set as fixed, then it is established as fixed, otherwise, the value of the leg's error is upgraded as:

$$
E r r_{l e g, j}=E r_{l e g, j} \pm \alpha_{2}\left(E_{l o s s, j}-E_{l o s s, a v g}\right)
$$

where $\pm \alpha_{2}$ is a factor for the value of switching losses, $E_{l o s s, j}$ is the total accumulated loss in the $j^{\text {th }}$ leg, and $E_{\text {loss,avg }}$ is the average loss accumulated in all legs in the phase. The next step is the extension of the system's model which supplies a significant method to control the loss dispensed between the whole of the switches of the converter. Total loss for each diode that starts conducting during the next control period is updated as:

$$
E_{j, x, d}=E_{j, x, d}+T\left(V_{O N, d}\left|\hat{i}_{k}\right|+\hat{i}_{k}^{2} R_{O N, d}\right)
$$

where $E_{j, x, d}$ is the present value of the accumulated loss for the jth leg where $x$ represents either the upper or lower switching device in the leg, $V_{O N, s w}$ and, $V_{O N, d}$ is the ONstate voltage drop of the switching device and $R_{O N, s w}, R_{O N, d}$ is the ON-state resistance of the switching device. The CHB converter configurations are cell-based built, which include several dc-dc converters and $\mathrm{H}$-bridges. The cascaded h-bridge configuration individually prospers for the PV farms into the power grid, because many PV panels can be connected separately to each cell [19].

Therefore, the maximum power in each PV chain may be different. Hence, an important challenge for these systems is the injection of balanced energy to the power network in an unbalanced condition due to the nature of PV systems, that amount of power generation depends on the weather changing. In [20] a finite control set-MPC in an unbalanced energy production for a cascaded h-bridge converter in PV applications has been reported with a satisfying cost function to overcome this problem.

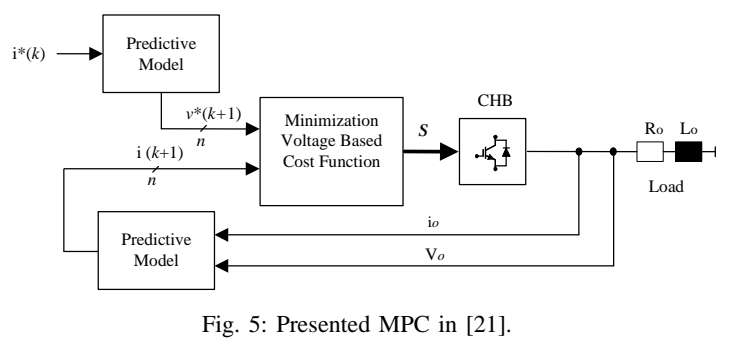

In the presented cost function, the problem of unbalanced energy generation is considered for the best control which consists of an input following error.

Furthermore, the predictive controller is formulated in the ABC-coordinate directly, to consider the common-mode voltage. By applying the transformation, the controller does the following on the balanced currents such as synthesizing an asymmetrical converter voltage and guarantees interface energy balancing. As a result, this predictive controller requires no online frame rotations. Therefore, this controller shows a simple construction and does not require any frame rotations while there is a reduction in series control steps. The conventional cost function of an FCS-MPC strategy by accounting the following input error is expressed as [21].

$$
J_{K}=\left\|i_{a b}^{\prime}(k+1)-i_{a b}^{*}(k+1)\right\|_{2}^{2}+\sigma\left\|u^{\prime}(k)+u^{*}(k)\right\|_{2}^{2}
$$

Fig. 5. displays the presented control strategy presented in [21]. In order to decrease the computation count of the CHB multilevel converter, a novel MPC strategy is reported in [22]. Consisting of three voltage level of the inverters, this strategy used by predicted voltage vectors that founded based on their references situations. For the optimal vector in the next stage, only the three closest vectors surrounding the triangular area are considered as the applicant when the triangular area is selected at each sampling step. This method has decreased the voltage vectors' applicant and computational 
count (regardless of the converter's level) due to the fact that only three surrounding vectors are applied. The voltage-based cost function to choose an optimal vector is expressed as :

$$
\left.g_{v}=\left(v_{\alpha}^{*}[k+1]-v_{\alpha}[k+1]\right)^{2}+v_{\beta}^{*}[k+1]-v_{\beta}[k+1]\right)^{2}
$$

An optimal vector and its switching state are found by repetitive computations.

The MPC method presented in [22] is exhibited in Fig. 6. In a $\mathrm{CHB}$ converter, the number of possible switching states will increase, by adding a number of cells which in turn, increases the computational burden.

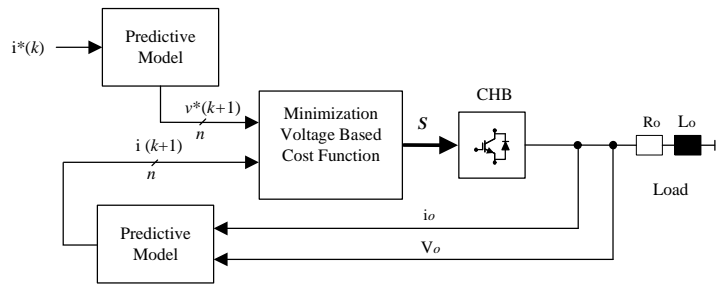

Fig. 6: Presented Model Predictive control in [22].

In [23], a new FCS-MPC approach is presented for a cascaded h-bridge multilevel converter which is a method based on a cell-by-cell algorithm. Namely, the sampling cycle $T s$ is separated into $n$ equal interruptions for an $n$-cell CHB converter. In the presented way to obtain these goals, the cost functions are expressed as:

$$
g_{i}=\left|i_{s}^{*}-i_{s}\right| \lambda_{v}\left|v_{C i}^{*}-v_{C i}\right|
$$

The flow diagram for this control method [23] is illustrated in Fig. 7. In this way, similar to the conventional FCS-MPC strategy, a two-step forward prediction has been considered. For compensation of the computation lag, the first stage is applied and the optimisation issue is resolved in the second stage.

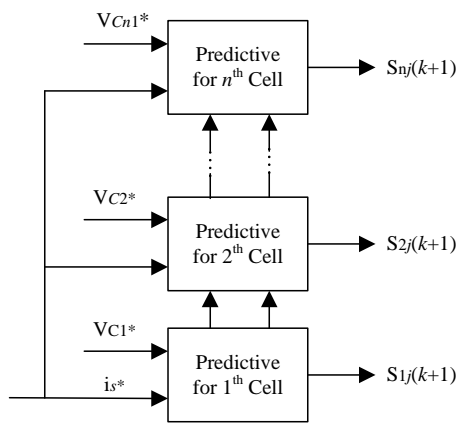

Fig. 7: Block diagram of cell-by-cell MPC control presented in [23].

In [24], a long prediction horizon FCS-MPC for CHBMLI is developed that considers, the whole of the steady-state system of the output voltages and currents in the general abccoordinate. Fig. 8 shows the block diagram of the presented method in [24]. In order to achieve a minimum common mode voltage (CMV), input references are designed. The presented cost function is:

$J_{N}=\sum_{l=k}^{k+N-1}\left\|i_{a b}(l+L)-i_{a b}^{*}(l+L)\right\|_{2}^{2}+\sigma\left\|u(l)-u^{*}(l)\right\|_{2}^{2}$

Here, $u(k)$ is the temporary control input that creates the current prediction $i_{a b}(k+1)$, and $u^{*}(k)$ represents the needed output voltages of the $\mathrm{CHB}$ in order to keep the current references at steady-state. Furthermore, to decrease the calculation a model predictive control it has changed into an equivalent optimisation issue that is resolved by the sphere decoding algorithm (SDA). According to this transformation, a graphical analysis of the recommended model predictive control was presented.

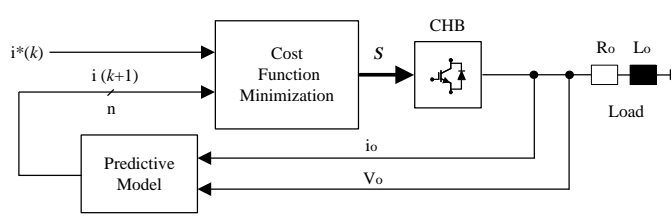

Fig. 8: MPC control strategy of presented in [24].

\section{DISCUSSION}

The model predictive control uses the model of the system to estimate the next behavior of variables at a time horizon. In the MPC system, the control action in a controller is executed by selecting online all possible switching states calculated in the discrete-time prediction model, so that the cost function is minimized. Therefore, with the proper formulation and designing a new cost function that includes all variables of the system, the flexibility function is possible. In this paper, we have evidenced the main nuances that have been documented by researchers which are, capacitor voltages balancing, optimisation of the switching capacitors, control of powers in STATCOM, reducing the computation burden.

Some of these papers worked on power control in STATCOM applications. In this way, in [13]-[17] by changing sampling times and considers capacitor voltages and load currents in the cost function, laminated optimisation process, current control and selection the best switching states to balance of the voltage's capacitors.

In a CHB converter, the number of possible switching states will increase by adding a higher quantity of cells. Research in [18]-[23]focused on the branch bound method, cell by cell algorithm in order to in order to reduce the CHB computation calculations. Finally, further investigations were conducted on long prediction horizon MPC by sphere decoding algorithm.

\section{CONCLUSION}

In this report, an overview of recent model predictive controls (MPC) applied to a CHB converter and its applications were presented. The research articles selected were extracted from the IEEE-explore database from the last couple of years. In order to fully comprehend the MPC strategy applied to CHB 
converters, the mathematical model of a three-phase CHB converter was explained. Then, the conventional MPC strategy normally applied to $\mathrm{CHB}$ converters was analyzed. The main objective behind this strategy radicated in the $\mathrm{CHB}$ converter was, the reduction of the computation burden, command mode voltage (CMV) as well as an in the grid-connected $\mathrm{CHB}$ converter such as STATCOM and PV systems, the control objectives were, the balancing of the capacitor voltage in each cell, the tracking of the reference current and the optimisation of the switching frequency.

\section{ACKNOWLEDGMENT}

The authors would like to thank the financial support of FONDECYT Regular 1160690 Research Project as well as MEC Project 80150056.

\section{REFERENCES}

[1] Y. Li and Z. Quan, "Derivation of multilevel voltage source converter topologies for medium voltage drives," Chinese Journal of Electrical Engineering, vol. 3, no. 2, pp. 24-31, September 2017.

[2] H. Samsami, A. Taheri, and R. Samanbakhsh, "New bidirectional multilevel inverter topology with staircase cascading for symmetric and asymmetric structures," IET Power Electronics, vol. 10, no. 11, pp. 1315-1323, 2017.

[3] L. Tan, B. Wu, M. Narimani, D. Xu, and G. Joos, "Multicarrierbased pwm strategies with complete voltage balance control for nnpc inverters," IEEE Transactions on Industrial Electronics, vol. PP, no. 99, pp. 1-1, 2017.

[4] Y. Deng, Y. Wang, K. H. Teo, M. Saeedifard, and R. G. Harley, "Optimized control of the modular multilevel converter based on space vector modulation," IEEE Transactions on Power Electronics, vol. PP, no. 99 , pp. 1-1, 2017.

[5] L. Tan, B. Wu, V. Sood, D. Xu, M. Narimani, Z. Cheng, and N. R. Zargari, "A simplified space vector modulation for four-level nested neutral-point clamped inverters with complete control of flying-capacitor voltages," IEEE Transactions on Power Electronics, vol. 33, no. 3, pp. 1997-2006, March 2018.

[6] N. B. A and P. Agarwal, "Nearest and non-nearest three vector modulations of npci using two-level space vector diagram-a novel approach," IEEE Transactions on Industry Applications, vol. PP, no. 99, pp. 1-1, 2017.

[7] M. Ahmed, A. Sheir, and M. Orabi, "Real-time solution and implementation of selective harmonic elimination of seven-level multilevel inverter," IEEE Journal of Emerging and Selected Topics in Power Electronics, vol. 5, no. 4, pp. 1700-1709, Dec 2017.

[8] J. I. Leon, S. Vazquez, and L. G. Franquelo, "Multilevel converters: Control and modulation techniques for their operation and industrial applications," Proceedings of the IEEE, vol. 105, no. 11, pp. 2066-2081, Nov 2017.

[9] R. P. Aguilera, P. Acua, P. Lezana, G. Konstantinou, B. Wu, S. Bernet, and V. G. Agelidis, "Selective harmonic elimination model predictive control for multilevel power converters," IEEE Transactions on Power Electronics, vol. 32, no. 3, pp. 2416-2426, March 2017.

[10] O. Machado, P. Martn, F. J. Rodrguez, and E. J. Bueno, "A neural network-based dynamic cost function for the implementation of a predictive current controller," IEEE Transactions on Industrial Informatics, vol. 13, no. 6, pp. 2946-2955, Dec 2017.

[11] M. Mosa, M. B. Shadmand, R. S. Balog, and H. A. Rub, "Efficient maximum power point tracking using model predictive control for photovoltaic systems under dynamic weather condition," IET Renewable Power Generation, vol. 11, no. 11, pp. 1401-1409, 2017.

[12] I. Jahn, C. D. Townsend, and H. Z. de la Parra, "Model-predictive modulation strategy for a hybrid si-sic cascaded h-bridge multi-level converter," in 2016 18th European Conference on Power Electronics and Applications (EPE'16 ECCE Europe), Sept 2016, pp. 1-10.

[13] Y. Zhang, X. Wu, X. Yuan, Y. Wang, and P. Dai, "Fast model predictive control for multilevel cascaded h-bridge statcom with polynomial computation time," IEEE Transactions on Industrial Electronics, vol. 63, no. 8, pp. 5231-5243, Aug 2016.
[14] Y. Zhang, X. Wu, and X. Yuan, "A simplified branch and bound approach for model predictive control of multilevel cascaded h-bridge statcom," IEEE Transactions on Industrial Electronics, vol. 64, no. 10, pp. 76347644 , Oct 2017

[15] C. D. Townsend, R. A. Baraciarte, Y. Yu, D. Tormo, H. Z. de La Parra, G. D. Demetriades, and V. G. Agelidis, "Heuristic model predictive modulation for high-power cascaded multilevel converters," IEEE Transactions on Industrial Electronics, vol. 63, no. 8, pp. 5263-5275, Aug 2016.

[16] E. Behrouzian, M. Bongiorno, and R. Teodorescu, "Impact of switching harmonics on capacitor cells balancing in phase-shifted pwm-based cascaded h-bridge statcom," IEEE Transactions on Power Electronics, vol. 32, no. 1, pp. 815-824, Jan 2017.

[17] S. C. Ferreira, R. B. Gonzatti, R. R. Pereira, C. H. da Silva, L. E. B. da Silva, and G. Lambert-Torres, "Finite control set model predictive control for dynamic reactive power compensation with hybrid active power filters," IEEE Transactions on Industrial Electronics, vol. 65, no. 3, pp. 2608-2617, March 2018.

[18] Q. Wei, B. Wu, D. . Xu, and N. R. Zargari, "Minimization of filter capacitor for medium-voltage current-source converters based on natural sampling svm," IEEE Transactions on Power Electronics, vol. 33, no. 1, pp. 473-481, Jan 2018.

[19] B. G. Devi and M. Mahesh, "A brief survey on different multilevel inverter topologies for grid-tied solar photo voltaic system," in 2017 IEEE International Conference on Smart Energy Grid Engineering (SEGE), Aug 2017, pp. 51-55.

[20] R. P. Aguilera, P. Acuna, Y. Yu, G. Konstantinou, C. D. Townsend, B. Wu, and V. G. Agelidis, "Predictive control of cascaded h-bridge converters under unbalanced power generation," IEEE Transactions on Industrial Electronics, vol. 64, no. 1, pp. 4-13, Jan 2017.

[21] R. P. Aguilera and D. E. Quevedo, "Predictive control of power converters: Designs with guaranteed performance," IEEE Transactions on Industrial Informatics, vol. 11, no. 1, pp. 53-63, Feb 2015.

[22] I. Kim, R. Chan, and S. Kwak, "Model predictive control method for chb multi-level inverter with reduced calculation complexity and fast dynamics," IET Electric Power Applications, vol. 11, no. 5, pp. 784792, 2017.

[23] C. Qi, X. Chen, P. Tu, and P. Wang, "Cell-by-cell-based finite-control-set model predictive control for a single-phase cascaded h-bridge rectifier," IEEE Transactions on Power Electronics, vol. 33, no. 2, pp. 1654-1665, Feb 2018.

[24] R. Baidya, R. P. Aguilera, P. Acua, S. Vazquez, and H. d. T. Mouton, "Multistep model predictive control for cascaded h-bridge inverters: Formulation and analysis," IEEE Transactions on Power Electronics, vol. 33, no. 1, pp. 876-886, Jan 2018. 